\title{
Impact of Risankizumab on PASI90 and DLQI0/1 Duration in Moderate-to-Severe Psoriasis: A Post Hoc Analysis of Four Phase 3 Clinical Trials
}

\author{
Mark G. Lebwohl · Ahmed M. Soliman · Hongbo Yang (D) • \\ Jessie Wang · Kaitlin Hagan · Byron Padilla · Andreas Pinter
}

Received: August 30, 2021 / Accepted: November 30, 2021 / Published online: December 18, 2021

(C) The Author(s) 2021

\begin{abstract}
Introduction: Novel therapies have allowed psoriasis patients to achieve high levels of skin clearance and meaningful improvements in health-related quality of life measures; however, duration of these outcomes has not been evaluated. This study aimed to estimate the duration of Psoriasis Area and Severity Index (PASI) 90 and Dermatology Life Quality Index (DLQI) 0/1 among patients with moderate-to-severe psoriasis receiving risankizumab and other treatments.

Methods: Pooled data from four phase 3 randomized clinical trials of risankizumab were used to estimate the number and proportion of days with PASI90 and DLQIO/1 during the
\end{abstract}

Supplementary Information The online version contains supplementary material available at https:// doi.org/10.1007/s13555-021-00660-3.

M. G. Lebwohl

Icahn School of Medicine at Mount Sinai, New York, NY, USA

A. M. Soliman · B. Padilla

AbbVie Inc., North Chicago, IL, USA

H. Yang $(\bowtie) \cdot J$. Wang $\cdot$ K. Hagan

Analysis Group, Inc., Boston, MA, USA

e-mail: hongbo.yang@analysisgroup.com

A. Pinter

University Hospital Frankfurt am Main, Frankfurt am Main, Germany 1-year post-baseline period with an area-underthe-curve approach. Patients were classified into five cohorts on the basis of their treatment experience during the follow-up period: risankizumab (RISA) only, RISA followed by re-randomization to RISA or placebo (RISA and RISA/ $\mathrm{PBO}$ ), adalimumab (ADA) followed by re-randomization to ADA or RISA (ADA and ADA/ RISA), ustekinumab (UST) only, and placebo followed by risankizumab (PBO/RISA).

Results: A total of 2101 patients were included in this analysis. Mean age was 47.5 years, $70 \%$ were males, and mean duration since psoriasis diagnosis was 18.6 years. Patients treated with RISA only throughout the study period experienced the longest PASI90 [245.7 days (67\% over 1 year)] and DLQI0/1 [213.7 (59\%)] duration. Patients treated with PBO/RISA [156.8 (43\%)] and UST only [154.2 (42\%)] experienced the shortest PASI90 duration. Similarly, patients treated with $\mathrm{PBO} / \mathrm{RISA}$ experienced the shortest DLQIO/1 duration during the 52-week study period [90.5 (25\%)].

Conclusion: Patients with moderate-to-severe psoriasis treated with risankizumab exhibited longer durations of PASI90 and DLQI0/1 than patients treated with other therapies.

Trial Registration: ClinicalTrials.gov identifiers: UltIMMa-1 (NCT02684370), NCT02684357 (UltIMMa-2), IMMvent (NCT02694523), IMMhance (NCT02672852). 
Keywords: Dermatology Life Quality Index; Health-related quality of life; Psoriasis; Psoriasis Area and Severity Index; Psoriasis skin clearance; Risankizumab

\section{Key Summary Points}

\section{Why carry out this study?}

Improvements in Psoriasis Area and Severity Index (PASI) and Dermatology Life Quality Index (DLQI) scores are generally assessed at specific timepoints in clinical trials of psoriasis biologic therapies.

This post hoc analysis aimed to estimate the duration of PASI90 and DLQI in patients with moderate-to-severe psoriasis from four phase 3 clinical trials.

\section{What was learned from the study?}

Patients with moderate-to-severe psoriasis treated with risankizumab alone experienced the longest duration of PASI90 (245.7 days) and DLQIO/1 (213.7 days) compared with other therapies.

Risankizumab treatment maintains positive outcomes for a longer duration compared with other therapies for moderate-to-severe psoriasis.

\section{INTRODUCTION}

Psoriasis is a chronic, immune-mediated disease characterized by a combination of inflammation and epidermal thickening, resulting in thick, scaly skin patches, affecting approximately 3\% of the US population [1]. Patients with psoriasis experience itchy, painful skin lesions that can lead to substantial impairments in physical and psychosocial functioning, resulting in emotional distress, a sense of stigmatization, worry, and restrictions in social, recreation, and work activities [2-4].
The Psoriasis Area and Severity Index (PASI) is the most widely used assessment tool for measuring the severity of psoriasis in clinical trials [5]. Until recently, reductions in PASI score of $75 \%$ (PASI75) were used as a treatment goal. However, with the advent of new biologic therapies, reduction in PASI score of $90 \%$ or more (PASI90, also referred to as almost clear and clear skin) has increasingly become the target in treating psoriasis [6]. Due to the significant impact of psoriasis on patients' quality of life, objective improvements in PASI alone are not sufficient for evaluating treatment efficacy. Meaningful improvements in health-related quality of life (HRQoL) measures, such as the Dermatology Life Quality Index (DLQI) $[7,8]$, are also needed. In particular, the proportion of patients achieving a DLQI score of 0 or 1 (i.e., psoriasis has no effect on patients' quality of life) is commonly evaluated in clinical trials. Results from four phase 3 trials (UltIMMa-1, UltIMMa-2, IMMvent, IMMhance) of risankizumab, an anti-IL-23 antibody, have demonstrated greater improvement in PASI and DLQI for patients treated with risankizumab compared with patients treated with placebo or other biologic medications [9-11].

While the efficacy of different treatments in achieving high levels of skin clearance and positive HRQoL outcomes has been assessed at specific timepoints, the duration of these outcomes associated with different treatments has not been previously studied. This study used data from four phase 3 clinical trials to estimate the duration of PASI90 and DLQIO/1 achieved by patients with moderate-to-severe psoriasis receiving risankizumab and other treatments over the 1-year post-baseline period.

\section{METHODS}

\section{Data Source}

This retrospective study pooled data from all treatment arms of the four phase 3 randomized clinical trials of risankizumab: UltIMMa-1 (NCT02684370) [9], UltIMMa-2 (NCT02684357) [9], IMMvent (NCT02694523) [10], and IMMhance (NCT02672852) [11]. Ethics 
committee approval was not required for this post hoc analysis, which prospectively collected clinical trial data conducted by AbbVie, and there were no interactions with patients or new data collection involved. Institutional review board approval was obtained in all studies included in this analysis. The studies were performed in accordance with the Declaration of Helsinki 1964 and its later amendments, and informed consent was obtained from all participants of the studies. All four trials were conducted in patients with moderate-to-severe psoriasis, defined as having affected body surface area $\geq 10 \%$, PASI score $\geq 12$, and static Physician Global Assessment (sPGA) $\geq 3$. The total follow-up period for the UltIMMa-1, UltIMMa-2, and IMMhance trials was 52 weeks, while the total follow-up period for the IMMvent trial was 44 weeks.

In the UltIMMa-1 and UltIMMa-2 trials, patients were randomized 3:1:1 at baseline to risankizumab, ustekinumab, or placebo. Patients receiving placebo switched to risankizumab after the initial 16 weeks. In the IMMvent trial, patients were randomized 1:1 at baseline to risankizumab or adalimumab (weeks $0-16$ ). At week 16, patients initially randomized to adalimumab were switched to risankizumab if they did not achieve PASI50 at week 16, remained on adalimumab if patients achieved PASI90 at week 16 , or were re-randomized 1:1 to either risankizumab or adalimumab if patients achieved PASI50-89 at week 16. In the IMMhance trial, patients were randomized 4:1 to risankizumab or placebo. At week 28, patients initially randomized to risankizumab who achieved an sPGA score of 0 or 1 were rerandomized to either risankizumab or placebo; those with sPGA score $\geq 2$ continued on risankizumab. Patients initially randomized to placebo switched to risankizumab at week 16 .

\section{Study Cohorts}

Patients were classified into cohorts on the basis of their treatment and re-randomization experience during the phase 3 trials (Fig. 1). To avoid potential selection bias, patients who were rerandomized and assigned subsequent treatments based on initial treatment response were combined into the same study cohort by initial treatment. For instance, patients in the IMMhance trial who initially received risankizumab and were then re-randomized to risankizumab or placebo based on sPGA at week 28 were grouped in the same study cohort. The study cohorts included risankizumab (RISA only), risankizumab followed by re-randomization to risankizumab or placebo (RISA and RISA/ PBO), ustekinumab (UST only), adalimumab followed by re-randomization to adalimumab or risankizumab (ADA and ADA/RISA), and placebo followed by risankizumab (PBO/RISA).

\section{Study Measures}

The duration of PASI90 and DLQIO/1 was estimated over the 1-year follow-up period after treatment initiation in the risankizumab trials. Duration of PASI90 (or PASI90 days) and duration of DLQIO/1 (or DLQIO/1 days) was defined as the cumulative nonconsecutive periods when patients achieved PASI90 ( $\geq 90 \%$ reduction from baseline PASI score) [12] and DLQIO/1 (no effect on patient's life) $[13,14]$, respectively.

\section{Statistical Analyses}

\section{Baseline Characteristics}

Baseline characteristics, including demographics, disease characteristics, treatment history, medical history, baseline patient-reported outcomes (PROs), and sPGA, were summarized and compared across study cohorts using Wilcoxon rank sum tests for continuous variables and chisquare tests for categorical variables.

\section{Estimation of PASI90 and DLQIO/1 Days}

An area under the curve (AUC) approach was used to estimate PASI90 days and DLQIO/ 1 days by study cohort over the 1-year post-baseline period.

The proportion of patients achieving PASI90 over the study period was plotted at each visit with available data. Between visits, the proportion of patients achieving PASI90 was assumed to have a linear change (i.e., each observed data point was connected linearly) to form a "PASI90 


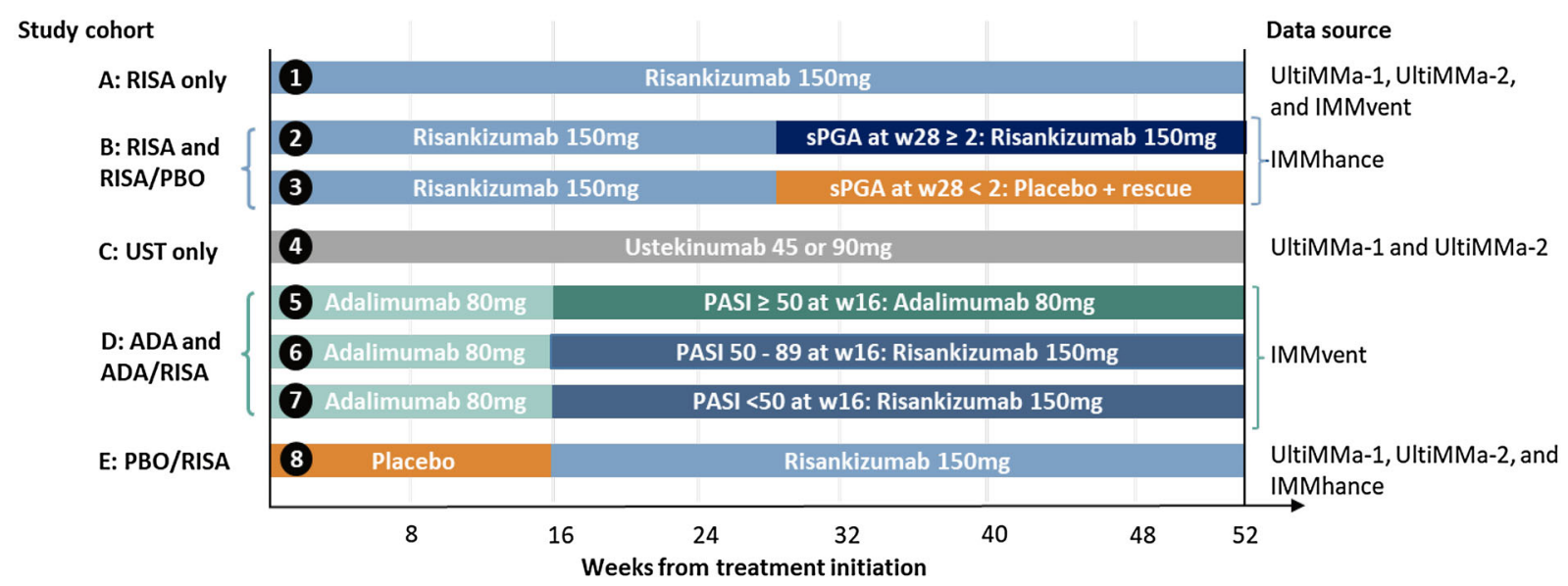

Fig. 1 Study cohort classification based on treatment and re-randomization experience during the phase 3 risankizumab trials. $A D A$ adalimumab, PASI Psoriasis Area and

curve." The area under the PASI90 curve represents the duration of PASI90 achieved by patients. For each study cohort, a curve was constructed and used to calculate the duration and percentage of PASI90 days over the study period. The number and proportion of days patients achieved a DLQI0/1 was estimated by study cohort based on the same AUC approach.

The AUC analyses accounted for all available PASI90 and DLQI0/1 measurements from the prespecified visits in the risankizumab trials. Weeks $0,4,8,12,16,20,24,28,32,34,36,40$, $44,46,48$, and 52 were included in the AUC analysis of PASI90, while weeks $0,12,16$, and 52 were included in the AUC analysis of DLQI0/1. At each timepoint, trials without prespecified PASI90 or DLQIO/1 data collection were excluded. For the IMMvent trial that ended at week 44, PASI score at week 52 was imputed based on the PASI score at week 44. The IMMhance trial did not collect DLQI data after week 16 and was thus excluded from the DLQIO/1 AUC analyses. Additionally, DLQI0/1 was not collected at week 12 in the IMMvent trial; therefore, the proportion of patients achieving DLQIO/1 at week 12 was estimated using data from the UltiMMa-1 and UltiMMa-2 trials only.

The primary AUC analyses of PASI90 days and DLQIO/1 days used modified nonresponder imputation (mNRI) to account for missing data at prespecified visits in the risankizumab trials.
Severity Index, $P B O$ placebo, RISA risankizumab, $s P G A$ static Physician Global Assessment, UST ustekinumab, $w$ week

The mNRI imputation definition was consistent with the definition used in the phase 3 risankizumab trials. At a visit with a missing outcome, data were imputed as failure for this and all subsequent visits if there were no data after the visit. If there were data available before and after the visit with a missing outcome, data were imputed as success if both the previous and subsequent visits were successes and data were imputed as failure if either the previous or subsequent visits were failures.

Sensitivity AUC analyses for PASI90 and DLQIO/1 days were conducted using last observation carried forward (LOCF) imputation to account for missing data at prespecified visits in the risankizumab trials.

\section{Conservative Scenario Analysis of PASI90 Days}

An additional conservative scenario analysis was conducted to estimate the duration of PASI90 days by study cohort. The number of days patients achieved PASI90 was calculated for each individual patient from baseline to week 52. The mNRI approach was used to account for missing PASI data at prespecified visits in the risankizumab trials. On days when PASI was not measured (i.e., between prespecified visits), patients' PASI score was assumed to remain the same as the prior observed PASI score. Since the IMMvent trial ended at week 
44 , it was assumed that patients remained at the week 44 PASI status for the remaining period up to week 52. After imputing the PASI score for each day during the 52-week trial follow-up period, the number of PASI90 days was calculated for each patient. The mean, standard deviation, and percentage of PASI90 days over the study period was estimated by study cohort.

\section{RESULTS}

\section{Baseline Characteristics}

A total of 2101 patients were included in this analysis. Patients were on average 47.5 years old, and the majority of patients were male $(70 \%)$ and white $(80 \%)$. The mean duration since psoriasis diagnosis was 18.6 years (Table 1). Proportion of males and mean BMI were comparable across study cohorts, although age, race, and ethnicity varied $(p<0.05)$. Patient- and physician-reported outcomes were also similar across study cohorts at baseline. Mean sPGA was higher than 3 for all cohorts, reflecting moderate-to-severe disease. Disease characteristics, treatment history, and comorbidities at baseline were consistent across cohorts, except for the prevalence of prior biologic therapy and psoriatic arthritis $(p<0.05)$.

\section{Analyses of PASI90 Days}

In the primary AUC analysis using mNRI-imputed PASI90, patients treated with RISA only throughout the study period experienced the longest PASI90 duration from baseline to week 52 [245.7 days ( $67 \%$ over 1 year) ], followed by patients who received RISA and RISA/PBO [228.5 days (63\% over 1 year)] and ADA and ADA/RISA [183.8 days (50\% over 1 year)] (Fig. 2). Patients who received PBO/RISA [156.8 days (43\% over 1 year)] and UST only [154.2 days ( $42 \%$ over 1 year)] had the shortest PASI90 durations from baseline to week 52 .

The sensitivity AUC analysis using LOCFimputed PASI90 resulted in comparable, though slightly larger, PASI90 duration estimates compared with the primary AUC analysis using
mNRI-imputed PASI90 (Supplementary Fig. 1). Patients treated with RISA only experienced the greatest number of days with PASI90 achieved from baseline to week 52 [253.0 days (69\% over 1 year)], and patients who received PBO/RISA [159.3 days (44\% over 1 year)] and UST only [157.3 days ( $43 \%$ over 1 year)] had the shortest PASI90 durations during the 52-week study period.

Similarly, in the conservative scenario analyses, patients who received RISA only throughout the study period had the longest PASI90 duration [242.3 days (66\% over 1 year)] (Supplementary Table 1 ). The AUC analysis, which assumed a linear change in the proportion of patients achieving PASI90, generally estimated slightly longer PASI90 durations compared with the results from the conservative scenario analysis.

\section{Analyses of DLQI0/1 Days}

In the primary AUC analysis using mNRI-imputed DLQIO/1, patients treated with RISA only throughout the study period experienced the longest DLQI0/1 duration from baseline to week 52 [213.7 days (59\% over 1 year)], followed by patients who received ADA and ADA/RISA [159.1 days (44\% over 1 year)], and UST only [144.3 days (40\% over 1 year)] (Fig. 3). Patients who received PBO/RISA had the shortest DLQIO/1 duration during the 52-week study period [ 90.5 days ( $25 \%$ over 1 year)].

The sensitivity AUC analysis using LOCFimputed DLQIO/1 resulted in consistent, though slightly larger, DLQI0/1 duration estimates compared with the primary AUC analysis that used mNRI-imputed DLQIO/1 (Supplementary Fig. 2). Patients who received RISA only experienced the longest DLQI0/1 duration (218.8 days [60\% over 1 year]), and patients who received $\mathrm{PBO} / \mathrm{RISA}$ had the shortest DLQIO/ 1 duration (91.8 days [25\% over 1 year]) during the 52-week study period.

\section{DISCUSSION}

This study utilized data from four phase 3 clinical trials to evaluate the impact of 
Table 1 Baseline characteristics across treatment cohorts of patients with moderate-to-severe psoriasis

\begin{tabular}{|c|c|c|c|c|c|c|c|}
\hline Patient Characteristics & $\begin{array}{l}\text { Overall } \\
\text { population } \\
N=2101\end{array}$ & $\begin{array}{l}\text { Cohort A } \\
\text { RISA only } \\
N=895\end{array}$ & $\begin{array}{l}\text { Cohort B } \\
\text { RISA and RISA/PBO } \\
N=406\end{array}$ & $\begin{array}{l}\text { Cohort C } \\
\text { ADA and ADA/RISA } \\
N=303\end{array}$ & $\begin{array}{l}\text { Cohort D } \\
\text { UST only } \\
N=197\end{array}$ & $\begin{array}{l}\text { Cohort E } \\
\text { PBO/RISA } \\
N=300\end{array}$ & $\begin{array}{l}\text { Global } \\
\text { test } p \text { - } \\
\text { value }^{\mathrm{a}}\end{array}$ \\
\hline \multicolumn{8}{|l|}{ Demographics } \\
\hline Age (years), mean $\pm S D$ & $47.5 \pm 13.5$ & $46.7 \pm 13.6$ & $49.6 \pm 13.2$ & $47.0 \pm 13.1$ & $47.6 \pm 14.2$ & $47.8 \pm 13.6$ & $0.006^{*}$ \\
\hline Male, $N(\%)$ & $\begin{array}{l}1469 \\
(69.9 \%)\end{array}$ & $622(69.5 \%)$ & $283(69.7 \%)$ & $211(69.6 \%)$ & $134(68.0 \%)$ & $219(73.0 \%)$ & 0.775 \\
\hline Race, $N(\%)$ & & & & & & & $0.016^{*}$ \\
\hline White & $\begin{array}{l}1681 \\
\quad(80.0 \%)\end{array}$ & $697(77.9 \%)$ & $319(78.6 \%)$ & $262(86.5 \%)$ & $163(82.7 \%)$ & $240(80.0 \%)$ & \\
\hline $\begin{array}{l}\text { Black or African } \\
\text { American }\end{array}$ & $62(3.0 \%)$ & $30(3.4 \%)$ & $18(4.4 \%)$ & $6(2.0 \%)$ & $3(1.5 \%)$ & $5(1.7 \%)$ & \\
\hline Asian & $327(15.6 \%)$ & $152(17.0 \%)$ & $64(15.8 \%)$ & $35(11.6 \%)$ & $26(13.2 \%)$ & $50(16.7 \%)$ & \\
\hline $\begin{array}{l}\text { American Indian or } \\
\text { Alaska Native }\end{array}$ & $16(0.8 \%)$ & $11(1.2 \%)$ & $0(0.0 \%)$ & $0(0.0 \%)$ & $2(1.0 \%)$ & $3(1.0 \%)$ & \\
\hline $\begin{array}{l}\text { Native Hawaiian or } \\
\text { other Pacific Islander }\end{array}$ & $8(0.4 \%)$ & $1(0.1 \%)$ & $3(0.7 \%)$ & $0(0.0 \%)$ & $2(1.0 \%)$ & $2(0.7 \%)$ & \\
\hline $\begin{array}{l}\text { Hispanic or Latino } \\
\text { ethnicity, } N(\%)\end{array}$ & $281(13.4 \%)$ & $111(12.4 \%)$ & $45(11.1 \%)$ & $59(19.5 \%)$ & $24(12.2 \%)$ & $42(14.0 \%)$ & $0.013^{*}$ \\
\hline $\begin{array}{l}\mathrm{BMI}\left(\mathrm{kg} / \mathrm{m}^{2}\right) \\
\quad \text { mean } \pm S D\end{array}$ & $30.6 \pm 7.1$ & $30.4 \pm 7.3$ & $31.3 \pm 7.3$ & $30.8 \pm 7.4$ & $30.4 \pm 6.9$ & $30.3 \pm 6.1$ & 0.303 \\
\hline \multicolumn{8}{|c|}{ Baseline disease characteristics } \\
\hline PASI, mean \pm SD & $20.0 \pm 7.6$ & $20.4 \pm 7.7$ & $19.9 \pm 7.9$ & $19.7 \pm 7.5$ & $19.1 \pm 6.4$ & $20.2 \pm 7.6$ & 0.177 \\
\hline $\begin{array}{l}\text { Duration since psoriasis } \\
\text { diagnosis (years), } \\
\text { mean } \pm S D\end{array}$ & $18.6 \pm 12.6$ & $18.1 \pm 12.5$ & $20.1 \pm 13.3$ & $18.2 \pm 12.3$ & $17.3 \pm 11.2$ & $19.1 \pm 12.7$ & 0.110 \\
\hline \multicolumn{8}{|l|}{ Treatment history } \\
\hline $\begin{array}{l}\text { Prior phototherapy/ } \\
\text { photochemotherapy, } \\
N(\%)\end{array}$ & $802(38.2 \%)$ & $356(39.8 \%)$ & $139(34.2 \%)$ & $122(40.3 \%)$ & $73(37.1 \%)$ & $112(37.3 \%)$ & 0.355 \\
\hline $\begin{array}{l}\text { Prior nonbiologic } \\
\text { systemic therapy, } N(\%)\end{array}$ & $\begin{array}{l}1017 \\
\quad(48.4 \%)\end{array}$ & $440(49.2 \%)$ & $191(47.0 \%)$ & $148(48.8 \%)$ & $101(51.3 \%)$ & $137(45.7 \%)$ & 0.721 \\
\hline $\begin{array}{l}\text { Prior biologic systemic } \\
\text { therapy, } N(\%)\end{array}$ & $885(42.1 \%)$ & $339(37.9 \%)$ & $230(56.7 \%)$ & $111(36.6 \%)$ & $72(36.5 \%)$ & $133(44.3 \%)$ & $<0.001^{*}$ \\
\hline $\begin{array}{l}\text { Naiive to all psoriasis } \\
\text { therapy (other than } \\
\text { topical), } N(\%)\end{array}$ & $405(19.3 \%)$ & $171(19.1 \%)$ & $69(17.0 \%)$ & $64(21.1 \%)$ & $36(18.3 \%)$ & $65(21.7 \%)$ & 0.514 \\
\hline \multicolumn{8}{|c|}{ Baseline patient-reported outcomes } \\
\hline $\begin{array}{l}\text { PSS total score } \\
\text { mean } \pm S D\end{array}$ & $8.2 \pm 3.7$ & $8.1 \pm 3.8$ & - & - & $8.5 \pm 3.7$ & $8.1 \pm 3.5$ & 0.481 \\
\hline DLQI, mean \pm SD & $13.3 \pm 7.1$ & $13.6 \pm 7.2$ & $13.1 \pm 7.0$ & $13.1 \pm 7.2$ & $12.7 \pm 7.0$ & $13.3 \pm 6.8$ & 0.541 \\
\hline $\begin{array}{l}\text { WLQ at-work } \\
\text { productivity } \\
\text { loss score }{ }^{c} \text {, mean } \pm S D\end{array}$ & $5.3 \pm 5.3$ & $5.3 \pm 5.1$ & - & $5.4 \pm 5.6$ & - & - & 0.847 \\
\hline Pain $V A S^{\mathrm{d}}$, mean $\pm S D$ & $40.8 \pm 30.6$ & $40.2 \pm 31.0$ & $47.0 \pm 30.1$ & - & $41.6 \pm 30.3$ & $36.0 \pm 30.1$ & 0.251 \\
\hline $\operatorname{PtGA} V A S^{\mathrm{c}}$, mean $\pm S D$ & $45.5 \pm 29.9$ & $46.6 \pm 30.4$ & $52.1 \pm 30.6$ & - & $38.1 \pm 28.9$ & $41.6 \pm 28.2$ & 0.118 \\
\hline \multicolumn{8}{|c|}{ Baseline physician-reported outcomes } \\
\hline $\mathrm{sPGA}^{\mathrm{f}}$, mean $\pm \mathrm{SD}$ & $3.2 \pm 0.4$ & $3.2 \pm 0.4$ & $3.2 \pm 0.4$ & $3.2 \pm 0.4$ & $3.2 \pm 0.4$ & $3.2 \pm 0.4$ & 0.775 \\
\hline \multicolumn{8}{|l|}{ Key comorbidities } \\
\hline $\begin{array}{l}\text { Psoriatic arthritis } \\
\text { (diagnosed or } \\
\text { suspected), } N(\%)\end{array}$ & $571(27.2 \%)$ & $215(24.0 \%)$ & $142(35.0 \%)$ & $62(20.5 \%)$ & $50(25.4 \%)$ & $102(34.0 \%)$ & $<0.001^{*}$ \\
\hline Hypertension, $N(\%)$ & $683(32.6 \%)$ & $273(30.6 \%)$ & $149(36.8 \%)$ & $101(33.4 \%)$ & $71(36.4 \%)$ & 89 (29.7\%) & 0.113 \\
\hline
\end{tabular}


Table 1 continued

\begin{tabular}{|c|c|c|c|c|c|c|c|}
\hline $\begin{array}{l}\text { Patient } \\
\text { Characteristics }\end{array}$ & $\begin{array}{l}\text { Overall } \\
\text { population } \\
N=2101\end{array}$ & $\begin{array}{l}\text { Cohort A } \\
\text { RISA } \\
\text { only } \\
N=895\end{array}$ & $\begin{array}{l}\text { Cohort B } \\
\text { RISA and RISA/PBO } \\
N=406\end{array}$ & $\begin{array}{l}\text { Cohort C } \\
\text { ADA and ADA/RISA } \\
N=303\end{array}$ & $\begin{array}{l}\text { Cohort D } \\
\text { UST only } \\
N=197\end{array}$ & $\begin{array}{l}\text { Cohort E } \\
\text { PBO/RISA } \\
N=300\end{array}$ & $\begin{array}{l}\text { Global } \\
\text { test } p \text {-value }\end{array}$ \\
\hline $\begin{array}{l}\text { Hyperlipidemia, } \\
N(\%)\end{array}$ & $490(23.4 \%)$ & $\begin{array}{l}199 \\
\quad(22.3 \%)\end{array}$ & $104(25.7 \%)$ & $60(19.8 \%)$ & $52(26.5 \%)$ & $75(25.2 \%)$ & 0.231 \\
\hline $\begin{array}{l}\text { Diabetes mellitus, } \\
\quad N(\%)\end{array}$ & $326(15.5 \%)$ & $\begin{array}{l}134 \\
(15.0 \%)\end{array}$ & $69(17.0 \%)$ & $50(16.5 \%)$ & 27 (13.7\%) & $46(15.3 \%)$ & 0.804 \\
\hline Obesity, $N(\%)$ & $1028(48.9 \%)$ & $\begin{array}{l}431 \\
\quad(48.2 \%)\end{array}$ & $206(50.7 \%)$ & $147(48.5 \%)$ & 92 (46.7\%) & $152(50.7 \%)$ & 0.823 \\
\hline
\end{tabular}

ADA: adalimumab; BMI: body mass index; DLQI: Dermatology Life Quality Index; PASI: Psoriasis Area and Severity Index; PBO: placebo; PRO: patientreported outcome; PSS: Psoriasis Symptom Scale; PtGA: Patient's Global Assessment; RISA: risankizumab; SD: standard deviation; sPGA: static Physician Global Assessment; UST: ustekinumab; VAS: visual analog scale; WLQ: Work Limitations Questionnaire

* Indicates statistical significance $(\mathrm{p}<0.05)$

- Indicates situations in which no patients in a treatment cohort had the PRO of interest reported

${ }^{a}$ Statistical comparisons were conducted using Kruskal-Wallis test for continuous variables and Chi-square test for categorical variables, unless frequency was $\leq 5$, in which case Fisher's exact test was used

${ }^{\mathrm{b}}$ PSS is a 4-item PRO instrument that was used to assess the severity of psoriasis symptoms in patients with moderate-to-severe psoriasis. The symptoms included are: pain, redness, itching, and burning from psoriasis. Current symptom severity was assessed as a daily diary, using a 5-point Likert-type scale ranging from 0 (none) to 4 (very severe)

'WLQ is a 25 -item questionnaire that was used to measure the degree to which health problems interfere with specific aspects of job performance and the associated health-related productivity loss. The WLQ has four scales: time management, physical demands, mental-interpersonal demands, and output demands. Item scores range from 0 (limited none of the time) to 4 (limited all of the time). Each scale is scored separately and scale scores are converted mathematically to 0 (no limitations) and 100 (most limitations). WLQ was only measured among patients in the IMMvent trial

${ }^{\mathrm{d}}$ Pain VAS is a visual analog scale that was used as an assessment of patient pain. The patient's assessment of pain was performed using a horizontal $10 \mathrm{~cm}$ VAS, ranging from 0 (no pain) to 100 (severe pain) after the question: "How much pain have you had because of your psoriatic arthritis in the past week?" ${ }^{\mathrm{e}} \mathrm{PtGA}$ of skin pain was used to assess the worst skin pain and the average skin pain due to psoriatic arthritis. Rating for the two items ranged from 0 (no skin pain) to 10 (skin pain as bad as you can imagine)

${ }_{\mathrm{f}} \mathrm{PGA}$ is a 5 point score ranging from 0 to 4 , based on the physician's assessment of the average thickness, erythema, and scaling of all psoriatic lesions. A lower score indicates less body coverage, with 0 being clear and 1 being almost clear

risankizumab and other therapies on the duration of almost clear or clear skin and achieving DLQI0/1. Using a novel AUC approach, this study was able to longitudinally measure PASI90 and DLQI0/1, which have previously only been described as cross-sectional measures of efficacy. Overall, patients with moderate-tosevere psoriasis treated with risankizumab throughout the study period consistently exhibited and maintained skin clearance and positive quality of life at longer durations compared to patients treated with other biologic therapies.

Prior studies have found that patients treated with risankizumab are more likely to achieve PASI90 and DLQIO/1 compared with other treatments. In a network meta-analysis of 41 randomized clinical trials, the short-term effectiveness of different biologic treatments for patients with moderate-to-severe psoriasis was evaluated [15]. This network meta-analysis found that significantly more patients treated with risankizumab achieved PASI90 over the short term (10-16 weeks) compared with patients treated with secukinumab, infliximab, ustekinumab, or adalimumab. In addition, another network meta-analysis compared PASI outcomes for different biologic therapies after 1 year of treatment [16]. This study concluded that treatment with risankizumab resulted in a higher probability of achieving PASI90 after 1 year of treatment compared with all other biologics. However, while greater, the probability of achieving PASI90 when comparing risankizumab with brodalumab and guselkumab was not significantly different. A third meta-analysis of 60 clinical trials also found risankizumab, brodalumab, guselkumab, and ixekizumab to be associated with the highest PASI response rates for both short-term and long-term therapy [17]. Finally, a network metaanalysis that compared and summarized the short-term efficacy and safety of IL-23 targeted drugs for the treatment of moderate-to-severe psoriasis found that patients treated with risankizumab had the greatest probability of 


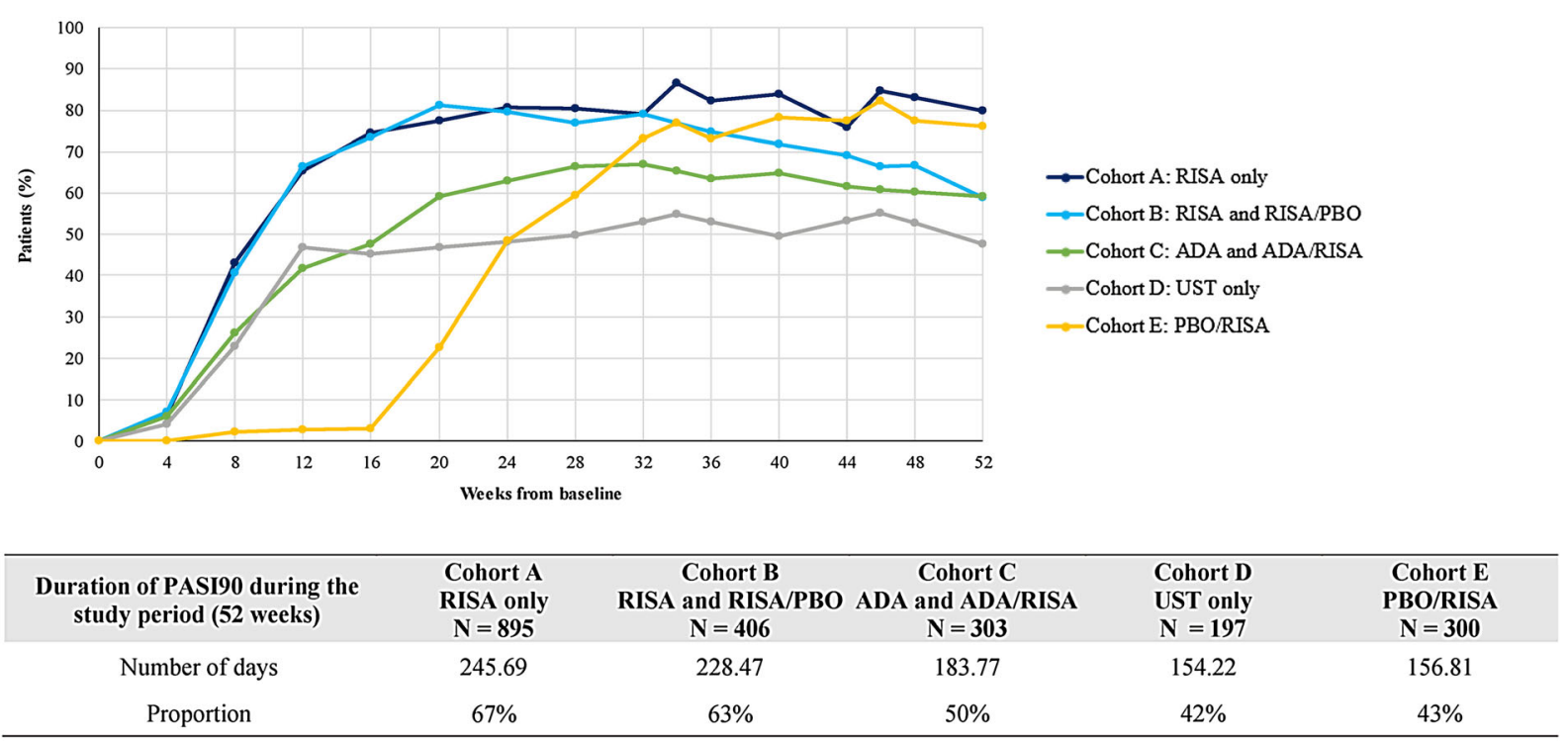

Fig. 2 Primary AUC analysis of PASI90 across study cohorts using mNRI imputation. ADA adalimumab, PASI Psoriasis Area and Severity Index, PBO placebo, RISA risankizumab, UST ustekinumab

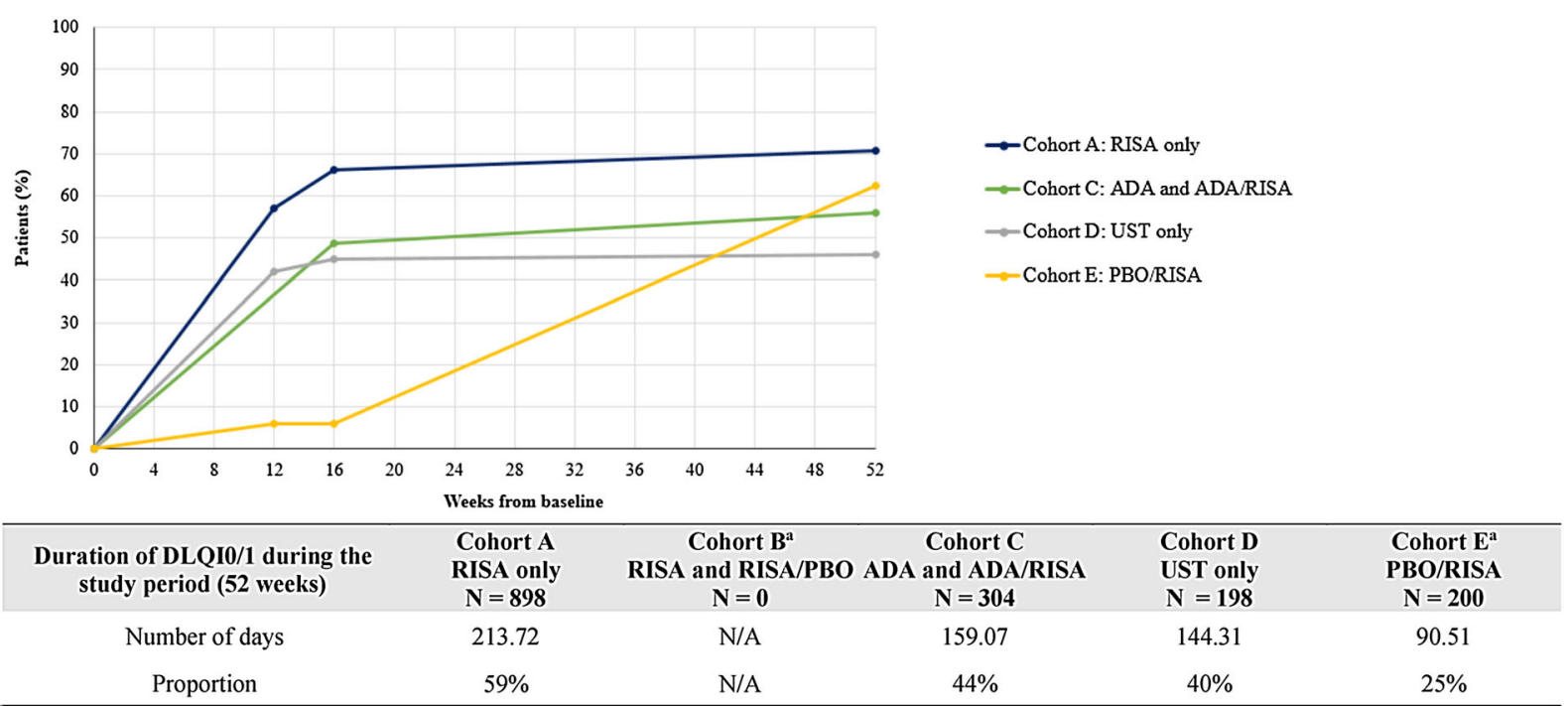

Fig. 3 Primary AUC analysis of DLQI0/1 across study cohorts using mNRI imputation. $A D A$ adalimumab, $D L Q I$ Dermatology Life Quality Index, $P B O$ placebo, RISA risankizumab, UST ustekinumab. ${ }^{\text {a}}$ Patients from

achieving DLQI0/1 compared with patients treated with ustekinumab, guselkumab, and tildrakizumab [18].
IMMhance trial did not collect DLQI data after week 16 and thus were excluded from the analysis. This resulted in exclusion of cohort B: RISA and RISA/PBO and a smaller sample of cohort $\mathrm{E}$

Robust and durable responses with risankizumab have also been demonstrated outside the clinical trial setting. A retrospective analysis of 154 patients with moderate-to-severe plaque 
psoriasis from multiple centers in the Czech Republic who were treated with risankizumab and followed up to 1 year found that PASI90 and PASI100 were achieved by $63.8 \%$ and $44.7 \%$ of patients, respectively, at week 16 and $82.4 \%$ and $67.6 \%$ of patients, respectively, at week 52 [19]. This trend was accompanied by an improvement in life quality, as reflected by a decline in DLQI over time. Moreover, no new safety signals were reported. In another multicenter study that was conducted in Italy, 57 adult patients with moderate-to-severe psoriasis were treated with risankizumab and followed up to 1 year; of the 55 patients $(96.5 \%)$ who completed the study, $85.5 \%$ and $60.0 \%$ achieved PASI90 and PASI100, respectively, at week 52 [20]. Taken together, these results provide realworld evidence for the high and sustained efficacy of risankizumab-comparable to that observed in clinical trials-in the treatment of psoriasis.

The present analysis demonstrates that patients treated with risankizumab not only achieve PASI90 and DLQI0/1 but are also more likely to have a longer duration of these outcomes. This study has several strengths, including the use of clinical trial data, which ensures that patients were closely monitored and followed for a significant period of time, and that the PASI and DLQI scores were well measured. In addition, different approaches (AUC and conservative scenario analysis) and missing data imputation assumptions (mNRI and LOCF) used to assess duration of almost clear skin or clear skin (PASI90) and DLQIO/1 showed consistent and robust results.

\section{Limitations}

Several limitations should be considered when interpreting the results of this study. As with all trial-based analyses, the results of this study may not be generalizable to a real-world population as the study population was a group of patients selected based on specific inclusion and exclusion criteria and followed under wellcontrolled conditions. Secondly, the study pooled patients across multiple trials on the basis of treatment experience, which could interfere with the randomization of the original trials. Breaking the randomization could result in unbalanced patient characteristics between study cohorts and introduce bias to the study findings. Finally, elements of study design prevented pooling certain treatment arms. For example, the risankizumab-only arm in the IMMhance study could not be pooled with risankizumab-only arms from the other trials, as these patients experienced re-randomization and only patients with worse sPGA remained on risankizumab after week 16.

\section{CONCLUSIONS}

Using a novel AUC approach, this study evaluated the duration of skin clearance and HRQoL for psoriasis patients treated with risankizumab and other therapies. Patients treated with risankizumab throughout the study period consistently exhibited and maintained skin clearance and positive quality of life at longer durations compared with patients treated with other biologic therapies. Overall, these findings highlight the benefit of risankizumab treatment for patients with moderate-to-severe psoriasis.

\section{ACKNOWLEDGEMENTS}

Funding. Funding for this study and the journals Rapid Service Fee was provided by AbbVie. The funders participated in the design and conduct of the study; collection, management, analysis, interpretation of data; preparation, review, or approval of the manuscript; and decision to submit the manuscript for publication.

Authorship. All named authors meet the International Committee of Medical Journal Editors (ICMJE) criteria for authorship for this article, take responsibility for the integrity of the work as a whole, and have given their approval for this version to be published.

Author Contributions. Dr(s) Yang, Wang, and Hagan had full access to all of the data in 
the study and take responsibility for the integrity of the data and the accuracy of the data analysis. Study concept and design: Soliman, Yang, Wang, Padilla. Acquisition, analysis, and interpretation of data: Lebwohl, Soliman, Yang, Wang, Hagan, Padilla, Pinter. Drafting of the manuscript: Hagan. Critical revision of the manuscript for important intellectual content: Lebwohl, Soliman, Yang, Wang, Hagan, Padilla, Pinter. Statistical analysis: Yang, Wang, Hagan. Obtained funding: Soliman, Padilla. Administrative, technical, or material support: Soliman, Padilla. Supervision: Soliman.

Medical Writing, Editorial and Other Assistance. The authors thank Sarah $\mathrm{H}$. Koenigsberg, an employee of Analysis Group, Inc., for medical writing assistance. This support was funded by AbbVie.

Disclosures. Mark G. Lebwohl is an employee of Mount Sinai and receives research funds from: Abbvie, Amgen, Arcutis, Avotres, Boehringer Ingelheim, Cara Therapeutics, Dermavant Sciences, Eli Lilly, Incyte, Janssen Research \& Development, LLC, Ortho Dermatologics, Regeneron, and UCB, Inc.; and is a consultant for Aditum Bio, Almirall, AltruBio Inc., AnaptysBio, Arcutis, Inc., Arena Pharmaceuticals, Aristea Therapeutics, Arrive Technologies, Avotres Therapeutics, BiomX, Brickell Biotech, Boehringer-Ingelheim, Bristol-Myers Squibb, Cara Therapeutics, Castle Biosciences, Corevitas, Dermavant Sciences, Dr. Reddy's Laboratories, Evelo Biosciences, Evommune, Inc., Facilitation of International Dermatology Education, Forte Biosciences, Foundation for Research and Education in Dermatology, Helsinn Therapeutics, Hexima Ltd., LEO Pharma, Meiji Seika Pharma, Mindera, Pfizer, Seanergy, and Verrica. Ahmed M. Soliman and Byron Padilla are employees of AbbVie who own AbbVie stock or stock options. Kaitlin Hagan, Jessie Wang, and Hongbo Yang are employed by Analysis Group, Inc., which received payment from AbbVie Inc. for participation in this research. Andreas Pinter was an investigator, speaker or advisor for AbbVie, Almirall-Hermal, Amgen, Biogen Idec, Biontec, Boehringer-Ingelheim, Celgene, GSK, Eli Lilly, Galderma,
Hexal, Janssen, LEO-Pharma, MC2, Medac, Merck Serono, Mitsubishi, MSD, Novartis, Pascoe, Pfizer, Tigercat Pharma, Regeneron, Roche, Sandoz Biopharmaceuticals, Sanofi-Genzyme, Schering-Plough and UCB Pharma.

Compliance with Ethics Guidelines. Ethics committee approval was not required for this post-hoc analysis, which prospectively collected clinical trial data conducted by AbbVie, Inc and there were no interactions with patients nor new data collection involved. Institutional review board approval was obtained in all studies included in this analysis. The studies were performed in accordance with the declaration of Helsinki 1964 and its later amendments and informed consent was obtained from all participants of the studies.

Data Availability. AbbVie is committed to responsible data sharing regarding the clinical trials we sponsor. This includes access to anonymized, individual and trial-level data (analysis data sets), as well as other information (e.g., protocols and Clinical Study Reports), as long as the trials are not part of an ongoing or planned regulatory submission. This includes requests for clinical trial data for unlicensed products and indications. This clinical trial data can be requested by any qualified researchers who engage in rigorous, independent scientific research, and will be provided following review and approval of a research proposal and Statistical Analysis Plan (SAP) and execution of a Data Sharing Agreement (DSA). Data requests can be submitted at any time and the data will be accessible for 12 months, with possible extensions considered. For more information on the process, or to submit a request, visit the following link: https://www.abbvie.com/ourscience/clinical-trials/clinical-trials-data-andinformation-sharing/data-and-informationsharing-with-qualified-researchers.html.

Open Access. This article is licensed under a Creative Commons Attribution-NonCommercial 4.0 International License, which permits any non-commercial use, sharing, adaptation, distribution and reproduction in any medium or format, as long as you give appropriate credit 
to the original author(s) and the source, provide a link to the Creative Commons licence, and indicate if changes were made. The images or other third party material in this article are included in the article's Creative Commons licence, unless indicated otherwise in a credit line to the material. If material is not included in the article's Creative Commons licence and your intended use is not permitted by statutory regulation or exceeds the permitted use, you will need to obtain permission directly from the copyright holder. To view a copy of this licence, visit http://creativecommons.org/licenses/by$\mathrm{nc} / 4.0 /$.

\section{REFERENCES}

1. Rachakonda TD, Schupp CW, Armstrong AW. Psoriasis prevalence among adults in the United States. J Am Acad Dermatol. 2014;70(3):512-6.

2. de Korte J, Sprangers MA, Mombers FM, Bos JD. Quality of life in patients with psoriasis: a systematic literature review. J Investig Dermatol Symp Proc. 2004;9(2):140-7.

3. Langley RG, Krueger GG, Griffiths CE. Psoriasis: epidemiology, clinical features, and quality of life. Ann Rheum Dis. 2005;64(Suppl 2):ii18-23.

4. Esposito M, Saraceno R, Giunta A, Maccarone M, Chimenti S. An Italian study on psoriasis and depression. Dermatology. 2006;212(2):123-7.

5. Mrowietz U, Kragballe K, Reich K, Spuls P, Griffiths CE, Nast A, et al. Definition of treatment goals for moderate to severe psoriasis: a European consensus. Arch Dermatol Res. 2011;303(1):1-10.

6. Papp KA, Blauvelt A, Bukhalo M, Gooderham M, Krueger JG, Lacour JP, et al. Risankizumab versus ustekinumab for moderate-to-severe plaque psoriasis. N Engl J Med. 2017;376(16):1551-60.

7. Finlay AY, Khan GK. Dermatology Life Quality Index (DLQI) - a simple practical measure for routine clinical use. Clin Exp Dermatol. 1994;19(3): 210-6.

8. Katugampola RP, Lewis VJ, Finlay AY. The Dermatology Life Quality Index: assessing the efficacy of biological therapies for psoriasis. $\mathrm{Br} \mathrm{J}$ Dermatol. 2007; 156(5):945-50.
9. Gordon KB, Strober B, Lebwohl M, Augustin M, Blauvelt A, Poulin Y, et al. Efficacy and safety of risankizumab in moderate-to-severe plaque psoriasis (UltIMMa-1 and UltIMMa-2): results from two double-blind, randomised, placebo-controlled and ustekinumab-controlled phase 3 trials. Lancet. 2018;392(10148):650-61.

10. Reich K, Gooderham M, Thaci D, Crowley JJ, Ryan C, Krueger JG, et al. Risankizumab compared with adalimumab in patients with moderate-to-severe plaque psoriasis (IMMvent): a randomised, doubleblind, active-comparator-controlled phase 3 trial. Lancet. 2019;394(10198):576-86.

11. Blauvelt A, Leonardi CL, Gooderham M, Papp KA, Philipp S, Wu JJ, et al. Efficacy and safety of continuous risankizumab therapy vs treatment withdrawal in patients with moderate to severe plaque psoriasis: a phase 3 randomized clinical trial. JAMA Dermatol. 2020;156(6):649-58.

12. Fredriksson T, Pettersson U. Severe psoriasis-oral therapy with a new retinoid. Dermatologica. 1978;157(4):238-44.

13. Mazzotti E, Barbaranelli C, Picardi A, Abeni D, Pasquini P. Psychometric properties of the Dermatology Life Quality Index (DLQI) in 900 Italian patients with psoriasis. Acta Derm Venereol. 2005;85(5):409-13.

14. Shikiar R, Willian MK, Okun MM, Thompson CS, Revicki DA. The validity and responsiveness of three quality of life measures in the assessment of psoriasis patients: results of a phase II study. Health Qual Life Outcomes. 2006;4:71.

15. Tada $Y$, Watanabe R, Noma H, Kanai $Y$, Nomura T, Kaneko K. Short-term effectiveness of biologics in patients with moderate-to-severe plaque psoriasis: a systematic review and network meta-analysis. J Dermatol Sci. 2020;99(1):53-61.

16. Yasmeen N, Sawyer LM, Malottki K, Levin LA, Didriksen Apol E, Jemec GB. Targeted therapies for patients with moderate-to-severe psoriasis: a systematic review and network meta-analysis of PASI response at 1 year. J Dermatolog Treat. 2020:1-15.

17. Armstrong AW, Puig L, Joshi A, Skup M, Williams $\mathrm{D}$, Li J, et al. Comparison of biologics and oral treatments for plaque psoriasis: a meta-analysis. JAMA Dermatol. 2020;156(3):258-69.

18. Shi J, Xu J, Chen Y. A network meta-analysis for the comparison of efficacy and safety of interleukin (IL)-23 targeted drugs in the treatment of moderate to severe psoriasis. Dermatol Ther. 2020:e13802.

19. Gkalpakiotis S, Cetkovska P, Arenberger P, Dolezal $\mathrm{T}$, Arenbergerova $\mathrm{M}$, Velackova $\mathrm{B}$, et al. 
Risankizumab for the treatment of moderate-tosevere psoriasis: real-life multicenter experience from the Czech Republic. Dermatol Ther. 2021;11(4):1345-55.
20. Hansel K, Zangrilli A, Bianchi L, Peris K, Chiricozzi A, Offidani A, et al. A 52-week update of a multicentre real-life experience on effectiveness and safety of risankizumab in psoriasis. J Eur Acad Dermatol Venereol. 2021. https://doi.org/10.1111/jdv. 17656. 